\title{
Corrigendum: Comparative Transcriptome, Metabolome, and Ionome Analysis of Two Contrasting Common Bean Genotypes in Saline Conditions
}

OPEN ACCESS

Approved by:

Frontiers Editorial Office, Frontiers Media SA, Switzerland

*Correspondence: Harun Niron

harun.niron@boun.edu.tr

Specialty section

This article was submitted to

Plant Abiotic Stress,

a section of the journal

Frontiers in Plant Science

Received: 19 May 2021

Accepted: 24 May 2021

Published: 29 June 2021

Citation:

Niron H, Barlas N, Salih B and Türet M (2021) Corrigendum: Comparative

Transcriptome, Metabolome, and Ionome Analysis of Two Contrasting

Common Bean Genotypes in Saline

Conditions.

Front. Plant Sci. 12:711806

doi: 10.3389/fpls.2021.711806
Harun Niron ${ }^{1 *}$, Nazire Barlas ${ }^{2}$, Bekir Salih $^{2}$ and Müge Türet ${ }^{1}$

${ }^{1}$ Department of Molecular Biology and Genetics, Bogazici University, Istanbul, Turkey, ${ }^{2}$ Department of Chemistry, Hacettepe University, Ankara, Turkey

Keywords: common bean, metabolome, Phaseolus vulgaris L., salt-stress, tolerance, transcriptome

\section{A Corrigendum on}

Comparative Transcriptome, Metabolome, and Ionome Analysis of Two Contrasting Common Bean Genotypes in Saline Conditions

by Niron, H., Barlas, N., Salih, B., and Türet, M. (2020). Front. Plant Sci. 11:599501. doi: 10.3389/fpls.2020.599501

In the original article there were mistakes in Figure 7, Supplementary Figure 4, and Additional File 8 as published.

The numbers defining the amount of sodium ion $\left(\mathrm{Na}^{+}\right)$in the root tissues of salt treated plants were erroneous by a factor of 10 . The corrected Figure 7, Supplementary Figure 4, and Additional File 8 appear.

The authors apologize for this error and state that this does not change the scientific conclusions of the article in any way. The original article has been updated.

Copyright $\odot 2021$ Niron, Barlas, Salih and Türet. This is an open-access article distributed under the terms of the Creative Commons Attribution License (CC BY). The use, distribution or reproduction in other forums is permitted, provided the original author(s) and the copyright owner(s) are credited and that the original publication in this journal is cited, in accordance with accepted academic practice. No use, distribution or reproduction is permitted which does not comply with these terms. 
A

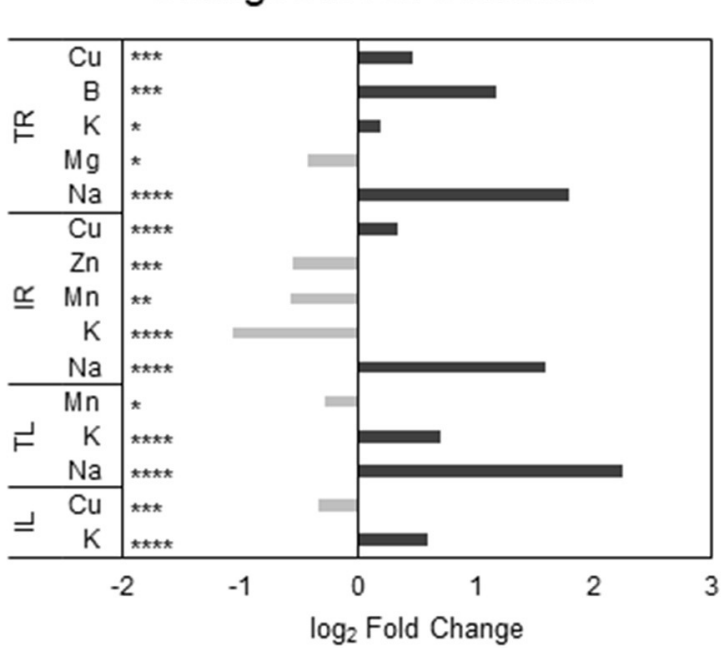

C

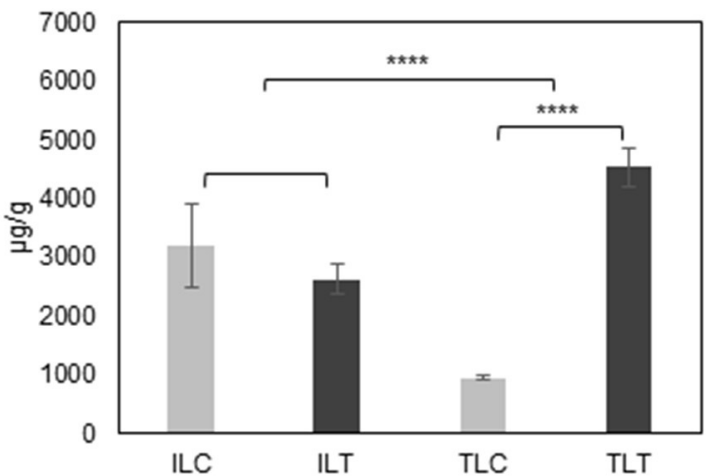

B

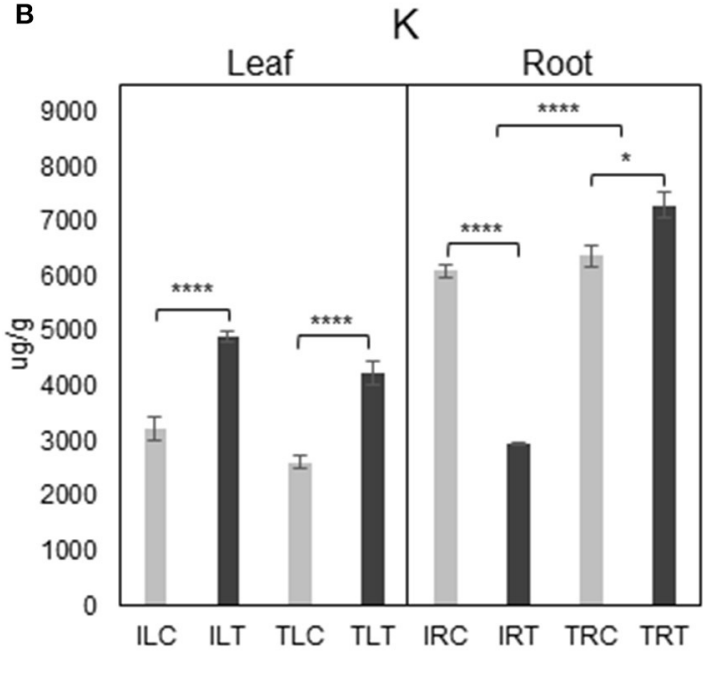

D

$\mathrm{Na}-\mathrm{Root}$

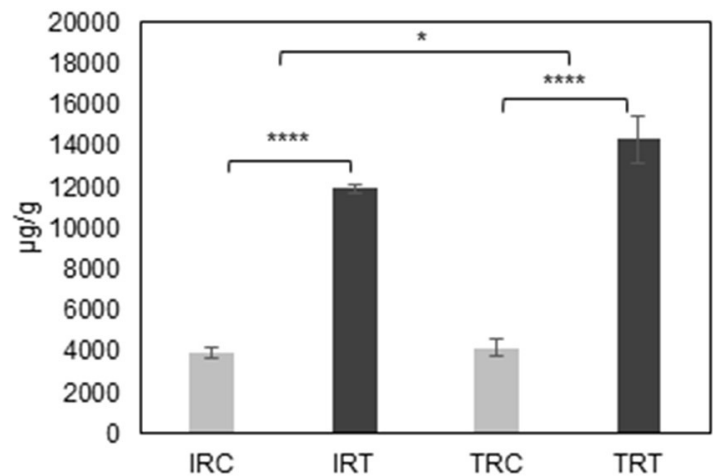

FIGURE 7 | Salt responsive ion content changes for tissues and genotypes (A). $\mathrm{K}^{+}$(B) and $\mathrm{Na}^{+}$(C,D) content changes were also displayed separately to emphasize the difference in changes between genotypes (C, control; T, salt treatment). Comparison of other ions can be found in Supplementary Figures 3, 4. * indicates significance and quantity of ${ }^{\star}$ displays the level of significance. $\left({ }^{*} p<0.05\right.$; ${ }^{\star \star} p<0.01$; $\left.{ }^{\star \star *} p<0.005 ;{ }^{\star \star \star *} p<0.001\right)$. 

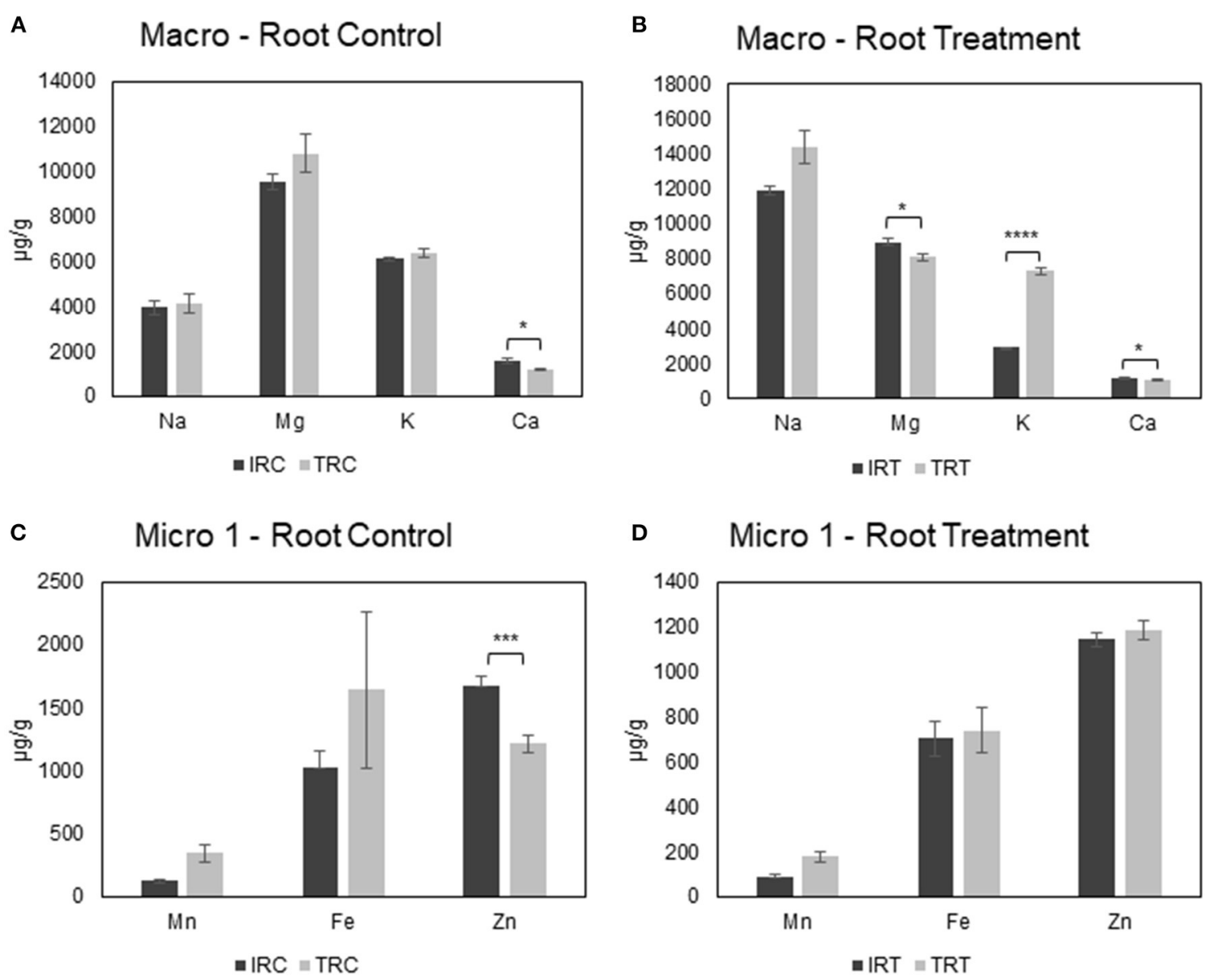

E Micro 2 - Root Control

F $\quad$ Micro 2 - Root Treatment
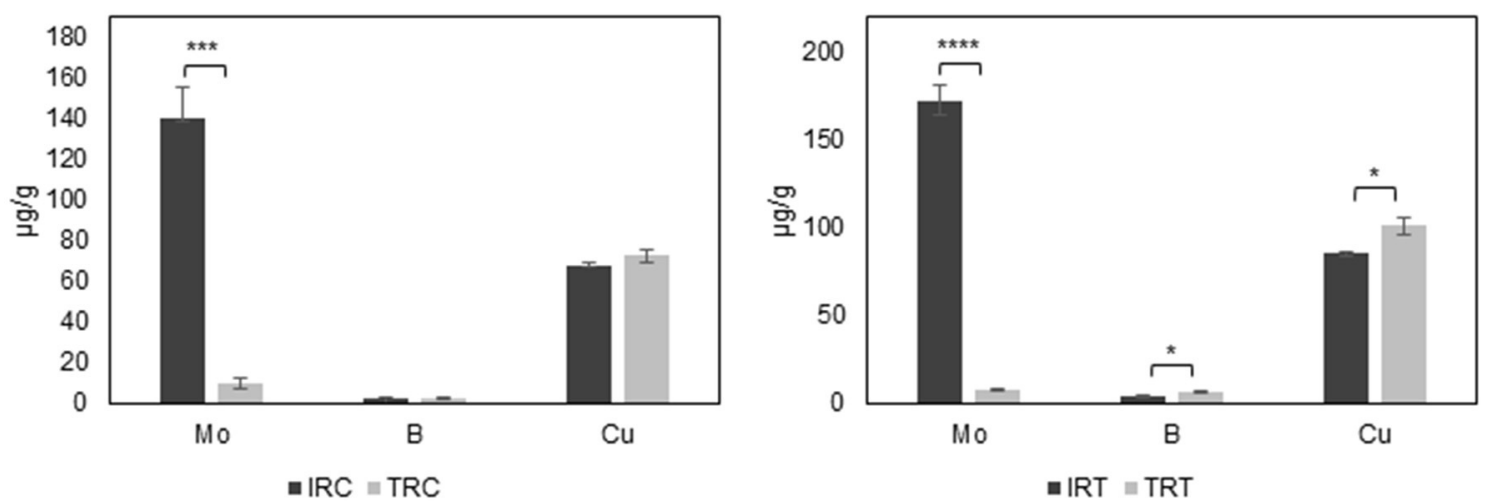

Supplementary Figure 4 | Comparison of root ion contents in control and saline conditions. (A,B) Display the macroelement content comparisons for control and treatment conditions respectively. (C-F) Display the microelement content comparisosn for control and treatment conditions respectively. ${ }^{*} p<0.05$; ${ }^{* *} p<0.01$; ${ }^{* * *} p<0.005 ;{ }^{* \star * *} p<0.001$ 
Additional File 8|

\begin{tabular}{|c|c|c|c|c|c|c|c|c|c|c|c|c|}
\hline Tissue/lon & \multicolumn{2}{|c|}{ ILC } & \multicolumn{2}{|c|}{ ILT } & \multicolumn{2}{|c|}{ TLC } & \multicolumn{2}{|c|}{ TLT } & \multicolumn{4}{|c|}{$p$-value (Condition or Genotype) } \\
\hline $\mathrm{Na}$ & $3,200.56$ & 711.50 & $2,630.80$ & 266.42 & 955.90 & 43.95 & $4,536.40$ & 318.10 & 0.531548 & 0.000470 & 0.047587 & 0.003630 \\
\hline $\mathrm{Mg}$ & $6,912.60$ & 423.75 & $6,978.20$ & 139.16 & $6,744.60$ & 294.62 & $6,403.20$ & 176.21 & 0.900617 & 0.405283 & 0.779220 & 0.052927 \\
\hline K & $3,220.40$ & 198.52 & $4,895.20$ & 99.95 & $2,604.20$ & 113.28 & 4,222.00 & 213.25 & 0.000556 & 0.000919 & 0.050198 & 0.045284 \\
\hline $\mathrm{Fe}$ & 115.99 & 12.89 & 83.34 & 14.14 & 103.94 & 13.12 & 85.26 & 8.73 & 0.165834 & 0.324456 & 0.574183 & 0.920976 \\
\hline $\mathrm{Zn}$ & 82.91 & 7.74 & 81.96 & 3.85 & 258.82 & 8.08 & 266.30 & 12.24 & 0.925291 & 0.662294 & 0.000001 & 0.000069 \\
\hline Mo & 13.11 & 1.50 & 10.42 & 0.43 & 1.21 & 0.21 & 1.24 & 0.13 & 0.186713 & 0.910741 & 0.001858 & 0.000013 \\
\hline B & 33.92 & 4.32 & 26.68 & 0.83 & 47.10 & 4.25 & 38.91 & 3.41 & 0.210658 & 0.217989 & 0.087716 & 0.030703 \\
\hline $\mathrm{Cu}$ & 8.44 & 0.30 & 6.67 & 0.12 & 9.58 & 0.92 & 7.04 & 0.20 & 0.003961 & 0.067807 & 0.340778 & 0.210593 \\
\hline $\mathrm{Na}$ & 3,933.20 & 286.98 & $1,1902.00$ & 213.90 & $4,146.00$ & 422.22 & $1,4354.76$ & 941.21 & 0.0000001 & 0.000137 & 0.720240 & 0.079241 \\
\hline $\mathrm{Mg}$ & $9,533.00$ & 373.79 & 8,969.80 & 217.32 & $1,0781.60$ & 854.08 & $8,088.40$ & 187.21 & 0.285407 & 0.046213 & 0.280206 & 0.025641 \\
\hline K & $6,098.80$ & 108.89 & $2,937.60$ & 23.38 & 6,368.60 & 202.81 & $7,293.00$ & 222.85 & 0.000007 & 0.025503 & 0.334053 & 0.000055 \\
\hline $\mathrm{Ca}$ & $1,548.20$ & 116.70 & $1,195.00$ & 27.86 & $1,173.40$ & 42.95 & $1,089.40$ & 24.16 & 0.052008 & 0.175876 & 0.042440 & 0.034105 \\
\hline $\mathrm{Mn}$ & 133.94 & 7.97 & 90.64 & 5.61 & 344.50 & 68.25 & 177.41 & 24.89 & 0.005084 & 0.094286 & 0.050369 & 0.033796 \\
\hline $\mathrm{Fe}$ & $1,031.80$ & 127.32 & 704.52 & 77.29 & $1,644.96$ & 619.72 & 740.70 & 98.48 & 0.092639 & 0.263811 & 0.431357 & 0.802912 \\
\hline $\mathrm{Zn}$ & $1,674.80$ & 80.12 & $1,143.40$ & 31.28 & $1,214.12$ & 64.93 & $1,184.20$ & 44.42 & 0.002360 & 0.743602 & 0.004328 & 0.522796 \\
\hline Mo & 140.04 & 15.41 & 171.90 & 8.35 & 10.14 & 2.53 & 7.63 & 1.02 & 0.153725 & 0.446274 & 0.001412 & 0.000051 \\
\hline B & 2.68 & 0.50 & 3.81 & 0.51 & 2.74 & 0.17 & 6.22 & 0.52 & 0.191954 & 0.002626 & 0.920176 & 0.018254 \\
\hline $\mathrm{Cu}$ & 67.14 & 1.55 & 85.11 & 0.89 & 72.44 & 3.28 & 100.76 & 4.56 & 0.000076 & 0.002522 & 0.241887 & 0.035853 \\
\hline
\end{tabular}

